\title{
Performance Analysis of Dual-Hop Multi-Relaying with Max-Mini Relay Selection over Composite Fading Channels
}

\author{
Chia-Hao Wu \\ Department of Communications, Navigation and \\ Control Engineering, WCR Lab., \\ National Taiwan Ocean University, \\ Pe-nin Road, Keelung, Taiwan, R.O.C. \\ e-mail: 10167023@ntou.edu.tw
}

\begin{abstract}
In dual-hop multi-relaying wireless systems, the non-closed form expression in log-normal probability distributions and high varying standard deviations makes it impossible to effectively execute performance analyses of outage probability and bit-error-rate (BER) performance levels; thus, an analysis framework was proposed for use in a composite (Rayleigh plus log-normal) fading channel. Developing analytical derivations involved generalizing the standard approaches for the probability density function (PDF) and the cumulative density function (CDF) of a composite fading channel at a conditioned constraint; that is, the signalto-noise power (SNR) of relay hop segments is relatively high to the targeted threshold when the max-mini relay selection scenario is adopted. The analytical deviations of outage probability and BER performance levels are closed-form expressions that are corroborated using Monte Carlo simulations and exact expression (integral form), yielding consistent results. Thus, the proposed analysis framework provides a simple and efficient approach for evaluating the influence of the shadowing effect and diversity order in system design applications.
\end{abstract}

Index Terms -amplify-and-forward (AF) relay, bit-error-rate (BER), cumulative density function (CDF), dual-hop multirelaying, log-normal fading, moment generation function (MGF), outage probability, probability density function (PDF), Rayleigh fading, standard deviation.

\section{INTRODUCTION}

Various analytical studies of relaying schemes [1 - 6] have focused on improving transmission throughput and reducing outage probability, concluding that amplify-and-forward (AF) relaying is the simplest and most economic relaying approach in practical deployment. Although the peak power constraint strategy is attractive [3], it is limited for realworld multi-relaying applications, because of the lack information on the log-normal shadowing effect. Analytical expressions for outage probability have been proposed based on imperfect non-identical log-normal fading channels [4] and the bit-error-rate (BER) performance levels have been analytically evaluated using cooperative relays that combine selections employing in-door log-normal fading for regenerative relay selection schemes [5]. Although log-normal fading has been used in previous studies, these have focused only on the channel state information (CSI) imperfection and the decode-and-forward

\author{
John F. An \\ Department of Communications, Navigation and \\ Control Engineering, WCR Lab., \\ National Taiwan Ocean University, \\ Pe-nin Road, Keelung, Taiwan, R.O.C. \\ e-mail: jfan@mail.ntou.edu.tw
}

(DF) relaying schemes. Numerous studies [6, 7] have approximated the log-normal distribution in a linear closedform or a more accurate expression, requiring complex algorithms and relay protocols. References [4, 7] modeled the power-sum of generically indoor correlated log-normal fading as a consequence of the particular structure of the end-to-end signal-to-noise power (SNR), conducting a performance analysis of multi-hop networks by using two receiver schemes. By resolving the end-to-end relaying lognormal sum-up using a Wilkinson approximation [4], probable closed-form expressions may be achieved in limited applications in which the in-door correlated lognormal fading channel is considered. Reference [8] employed a performance analysis using a closed form approach for a cooperative network; however, these expressions considered only Rayleigh fading and not the shadowing effect. We now consider the general case in which only the "best" relay was selected over multi-relay nodes to participate in signal forwarding, in the underlying source-relay-destination $(S \rightarrow R \rightarrow D)$ link by using the maxmini relay selection scenario $[2,8]$. Because the max-mini relay selection process has been extensively examined $[2,8$, 9], it was omitted from this paper. Thus, the minimum probability theory and maximum probability theory were used to generate the cumulative density functions (CDF) of the single dual-hop relaying and the CDF of dual-hop multirelaying links (i.e., $\mathrm{M}$ relay nodes), respectively. Moreover, the corresponding moment generating function (MGF) was derived to generate the analytical derivations of the outage probability and BER performance; the simulation results and exact solutions thereof were compared with the analytical deviations, indicating that the proposed closedforms approximations are highly accurate when a standard deviation constraint is imposed. The main contribution of this paper is refining a simple and effective analysis framework for used in a composite fading environment.

\section{SYSTEM MODELS AND CHANNEL STATISTICS}

Figure 1 shows a schematic of a dual-hop multi-relaying network that contains one source node $(\mathrm{S})$, one destination node (D), and $\mathrm{M}$ relay nodes (Rs). 


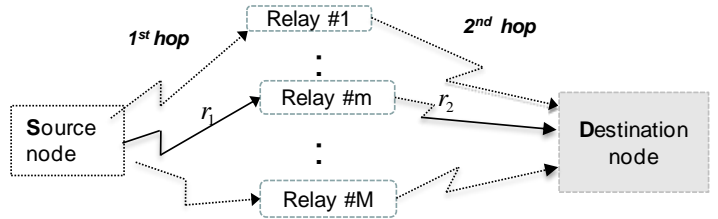

Fig. 1 Dual-hop multi-relaying system

The system model is represented by the following equations: $\gamma_{R}=r_{1} \cdot \widetilde{s}+n_{1}$

$\gamma_{D}=G \cdot r_{2}\left(\gamma_{R}\right)+n_{2}$

where $\gamma_{R}$ is the received signal at $\mathrm{R}, \gamma_{D}$ is the received signal at $\mathrm{D}$ with a relay gain $G$, and the channel gains are $r_{1}$ and $r_{2}$ for the first relay hop $\mathrm{S} \rightarrow \mathrm{R}$ and second hop $\mathrm{R} \rightarrow \mathrm{D}$, respectively. Only the "best" relay [2] was selected as a candidate to relay the signal, $\tilde{S}$, with signal power $E\left[\tilde{s} \widetilde{s}^{*}\right]=\mathcal{E}_{S}$ to $\mathrm{D}$ node. Numerous relay selection schemes have been extensively examined in [2, 8, 9]; however, these schemes therein are not included in the current study. The additive white Gaussian noise, $n_{i}$, for $i$ $=1$ and 2 at the first relay hop $\mathrm{S} \rightarrow \mathrm{R}$ and second hop $\mathrm{R} \rightarrow$ $\mathrm{D}$, respectively, are mutually independent with $n_{i} \sim$ $C \mathcal{N}\left(0, N_{0}\right)$. The relay amplification power factor is defined as $G^{2}=1 /\left(r_{1}^{2}+N_{0} / \mathcal{E}_{S}\right)[1,7]$, and the CSI was assumed to be accurately estimated. The channel gains, $r_{i}$, are Rayleigh fading comprising superimposed long-term log-normal variations (i.e., composite Rayleigh plus lognormal fading). Dual-hop relaying was primarily considered for a semi-asymmetric network in which relays are mid-way between the source and destination and normalized by path loss for each relaying link. Regarding the selected dual-hop relay, the instantaneous SNR is given as $\chi_{i}=\left|r_{i}\right|^{2} S N R_{0}$, where $i=1,2$ corresponds to the first and the second hops, respectively. By definition, the average SNR and the mean value are given as $S N R_{0}=\mathcal{E}_{S} / N_{0}$ and $\bar{\chi}_{i}=E\left[\chi_{i}\right]$ in regard to the $i$ th hop segment.

\section{A. Rayleigh Fading Statistics:}

For rapid short-term fading, the instantaneous power of a signal with Rayleigh distributed random variables shows that the PDF of $\chi_{i}$ is expressed as an exponential function on the $i$ th hop of an individual dual-hop relaying link:

$$
p_{\chi}\left(\chi_{i}\right)=\frac{1}{\bar{\chi}_{i}} \exp \left(-\chi_{i} / \bar{\chi}_{i}\right) \quad,, i=1,2 \text {. }
$$

The associated CDF in regard to a target threshold $\chi_{T}$ is given as follows:

$$
p_{\chi}\left(\chi_{i} \leq \chi_{T}\right)=1-\exp \left(-\chi_{T} / \bar{\chi}_{i}\right)
$$

When considering a conditioned constraint $\bar{\chi}_{i} \gg \chi_{T}$, the asymptotic expression of (3) is approximated as follows:

$\lim _{\bar{\chi}_{i} \rightarrow \infty} P_{\chi}\left(\chi_{i}<\chi_{T}\right)=\frac{\chi_{T}}{\bar{\chi}_{i}}$

The CDF of Rayleigh fading was simplified in this study.

\section{B. Log-normal Fading Statistics}

It is generally accepted that slow variation is log-normal distributed over the area mean. Regarding long-term averages of the composite fading channel, the shadowing mean is typically called the area mean. Thus, a local mean $\bar{\chi}_{i}$ fluctuates near the area mean $\zeta_{i}$, and based on the PDF of log-normal fading [11, p.48] for each relay hop,

$P_{l}\left(\bar{\chi}_{i}\right)=\frac{\kappa}{\sqrt{2 \pi} \sigma_{i} \cdot \bar{\chi}_{i}} \exp \left[\frac{-\left(\bar{\chi}_{i, d}-\varsigma_{i, d}\right)^{2}}{2 \sigma_{i}^{2}}\right] \quad, i=1,2$

where $\kappa=\log e$ and a subscript " $d$ " indicates that a signal is in logarithm; $\bar{\chi}_{i, d}=\log _{10}\left(\bar{\chi}_{i}\right), \varsigma_{i, d}=\log _{10}\left(\varsigma_{i}\right)$, and $\sigma_{i}$ denotes a standard deviation in logarithm.

\section{Composite Fading Statistics:}

When the short-term and log-term distributions are superimposed, the resulting marginal PDF $[11,12]$ for each relay hop segment is as follows:

$$
P_{c}\left(\chi_{i}\right)=\int_{0}^{\infty} P_{\chi}\left(\chi_{i} / \bar{\chi}_{i}\right) P_{l}\left(\bar{\chi}_{i}\right) d \bar{\chi}_{i}, \quad i=1,2
$$

Substituting (2) and (5) in (6), the PDF (6) is given by

$$
P_{c}\left(\chi_{i}\right)=\int_{0}^{\infty} \frac{1}{\bar{\chi}_{i}} \exp \left(\frac{-\chi_{i}}{\bar{\chi}_{i}}\right) \frac{\kappa}{\sqrt{2 \pi} \sigma_{i} \bar{\chi}_{i}} \exp \left[\frac{-\left(\bar{\chi}_{i, d}-\varsigma_{i, d}\right)^{2}}{2 \sigma_{i}^{2}}\right] d \bar{\chi}_{i},
$$

and the associated $\mathrm{CDF}$ in regard to the target threshold $\chi_{T}$ is given by

$P_{c}\left(\chi_{i} \leq \chi_{T}\right)=F_{\chi_{i}}\left(\chi_{T}\right)$

$=\int_{0}^{\infty}\left[1-\exp \left(-\chi_{T} / \bar{\chi}_{i}\right)\right] \cdot \frac{\kappa}{\sqrt{2 \pi} \sigma_{i} \bar{\chi}_{i}} \exp \left(\frac{-\left(\bar{\chi}_{i, d}-\varsigma_{i, d}\right)^{2}}{2 \sigma_{i}^{2}}\right) d \bar{\chi}_{i}, i=1, \quad 2$.

Under the conditioned constraint $\bar{\chi}_{i} \gg \chi_{T}$, by substituting (4) and (5) in (6) the CDF in regard to (8) can be rewritten as

$P_{c}\left(\chi_{i} \leq \chi_{T}\right)=\frac{\kappa \cdot \chi_{T}}{\sqrt{2 \pi} \sigma_{i}} \int_{0}^{\infty} \frac{1}{\bar{\chi}_{i}^{2}} \cdot \exp \left(\frac{-\left(\bar{\chi}_{i, d}-\varsigma_{i, d}\right)^{2}}{2 \sigma_{i}^{2}}\right) d \bar{\chi}_{i}, \quad i=1,2$.

The relationship between $\bar{\chi}_{i, d}$ and $\zeta_{i, d}$ (median value of $\chi_{i}$ ) is given as [11]

$\bar{\chi}_{i, d}=\varsigma_{i, d}+\frac{\sigma_{i}^{2}}{2 \cdot \kappa}$.

Setting the condition $\bar{\chi}_{i}=\varepsilon \cdot \varsigma_{i}$, yielded $\chi_{i, d}-\varsigma_{i, d}=\kappa \cdot \ln \varepsilon$, where $\ln$ is a natural $\log$ function, $1 / \bar{\chi}_{i} \cdot d \bar{\chi}_{i}=d(\ln \varepsilon)$ and $\varepsilon$ is an internal variable; thus, the CDF (9) was rewritten as follows: 


$$
\begin{aligned}
P_{c}\left(\chi_{i}<\chi_{T}\right) & =F_{\chi_{i}}\left(\chi_{T}\right) \\
& =\frac{\kappa \cdot \chi_{T}}{\sqrt{2 \pi} \sigma_{i}} \int_{0}^{\infty} \frac{1}{\varepsilon \cdot \varsigma_{i}} \cdot \exp \left(\frac{-(\ln \varepsilon)^{2} \cdot(\kappa)^{2}}{2 \sigma_{l}^{2}}\right) d(\ln \varepsilon)
\end{aligned}
$$

A closed-form expression was obtained as follows:

$$
F_{\chi_{i}}\left(\chi_{T}\right)=\frac{\chi_{T}}{\varsigma_{i}} \exp \left(\sigma_{i}^{2} / 2 \kappa^{2}\right) \text {. }
$$

Using the anti-log in (10), yielded $\varsigma_{i}=\bar{\chi}_{i} \cdot \exp \left(\frac{-\sigma_{i}^{2}}{2 \kappa^{2}}\right)$, which was substituted in (12) to derive the following:

$F_{\chi_{i}}\left(\chi_{T}\right)=\frac{\chi_{T}}{\bar{\chi}_{i}} \exp \left(\frac{\sigma_{i}^{2}}{\kappa^{2}}\right)$

and its corresponding PDF derives

$$
P_{c}\left(\chi_{i}\right)=\frac{1}{\bar{\chi}_{i}} \cdot \exp \left(\frac{\sigma_{i}^{2}}{\kappa^{2}}\right) \text {. }
$$

This equation is relevant to the log-normal standard deviation and the mean SNR value of the $i$ th hop component.

\section{OUtAge Probability}

The outage probability of dual-hop multi-relaying was accessed using the PDFs and CDFs presented in Section II. In the relaying application, the outage probability was defined as the probability that the system fails to support a target data rate, $\lambda$. This target rate corresponds to a specific SNR threshold $\left(\chi_{T}\right)$ for the system requirement. When considering a half-duplex operation for dual-hop relaying, this threshold relates to the target end-to-end data rate, $\lambda$ (bit/Hz/sec.) by using $\chi_{T}=2^{2 \lambda}-1$. Using a max-mini relay selection criterion, the outage probability was derived using the maximum and minimum probabilities [10] sequentially to select relays over $\mathrm{M}$ multi-relaying links and the hop selection over the dual hops, respectively, assuming that both relaying hops are mutually independent.

In the first stage, the CDF of each relaying link was calculated using the minimum probability [10] of $L_{m}=\min \left\{\chi_{1, m}, \chi_{2, m}\right\}, m=1,2, . ., M$ (i.e., the smallest $\chi_{i, m}$ is less than an arbitrary value, $\chi$ ).

$$
\begin{aligned}
F_{c}(\chi)= & 1-P_{c}\left(\chi_{1, m}>\chi\right) \cdot P_{c}\left(\chi_{2, m}>\chi\right) \\
& =1-\left[1-F_{\chi_{1, m}}(\chi)\right] \cdot\left[1-F_{\chi_{2, m}}(\chi)\right] \\
& =F_{\chi_{1, m}}(\chi)+F_{\chi_{2, m}}(\chi)-F_{\chi_{1, m}}(\chi) F_{\chi_{2, m}}(\chi)
\end{aligned}
$$

where $F_{\chi_{1, m}}(\chi)$ and $F_{\chi_{2, m}}(\chi)$ are the CDF of $\chi_{1, m}$ and $\chi_{2, m}$ in the first hop and the second hop, respectively. After Substituting (13) in (15) with $\chi_{T}=\chi$ and a proper index $i=$ 1 and 2 for both hop segments, (15) was rewritten as

$$
\begin{aligned}
F_{c}(\chi)= & \frac{\chi}{\bar{\chi}_{1, m}} \exp \left(\tilde{\sigma}_{1}^{2} / \tilde{\kappa}^{2}\right)+\frac{\chi}{\bar{\chi}_{2, m}} \exp \left(\tilde{\sigma}_{2}^{2} / \widetilde{\kappa}^{2}\right) \\
& -\frac{\chi^{2}}{\bar{\chi}_{1, m} \cdot \bar{\chi}_{2, m}} \exp \left(\frac{\tilde{\sigma}_{1}^{2}+\tilde{\sigma}_{2}^{2}}{\tilde{\kappa}^{2}}\right)
\end{aligned}
$$

where the standard deviations, $\tilde{\sigma}_{1}=10 \sigma_{1} \quad(\mathrm{~dB})$ and $\tilde{\sigma}_{2}=10 \sigma_{2}(\mathrm{~dB})$, correspond to the first and second hops, respectively, and $\tilde{\kappa}=10 \kappa$. This arrangement expressed the standard deviation in $\mathrm{dB}$ without losing generality. The PDF of $F_{c}(\chi), P_{c}(\chi)$ can be obtained by differentiating (16) in regard to $\chi$.

In the second stage, the CDF of the relay selection was accessed for the M multi-relaying link, where the maximum probability [11] is employed for the " best" relay selection of $\chi_{b}=\max \left\{L_{m}\right\}, m=1,2, \ldots, M$. Because the largest instantaneous SNR yields $\chi_{i}=\chi_{b}$, the CDF over M relay nodes can be express using [13]:

$F_{C}(\chi)=\left[F_{c}(\chi)\right]^{M}$.

The corresponding PDF can be obtained using the derivative of (17) in regard to $\chi$,

$P_{C}(\chi)=M \cdot p_{c}(\chi) \cdot\left[F_{c}(\chi)\right]^{M-1}$.

Thus, the outage probability for multi-relaying can be expressed using

$$
\begin{aligned}
P_{\text {out }} & =\int_{0}^{\chi_{T}} P_{C}(\chi) d \chi \\
& =\left[F_{c}\left(\chi_{T}\right)\right]^{M}
\end{aligned}
$$

where $\chi_{T}$ is the SNR target threshold. After substituting (16) in (19) and applying algebraic manipulations, the outage probability can be expressed in binomial form:

$$
\begin{aligned}
P_{\text {out }} & =\left(\mathrm{A} \chi_{T}-\mathrm{B} \chi_{T}\right)^{M} \\
& =\sum_{l=0}^{M}(-1)^{M}\left(\begin{array}{l}
M \\
l
\end{array}\right) \mathrm{A}^{M-l} \mathrm{~B}^{l} \chi_{T}^{M+l}
\end{aligned}
$$

where $\mathrm{A}=\frac{1}{\bar{\chi}_{1, m}} \cdot \exp \left(\frac{\tilde{\sigma}_{1}^{2}}{\tilde{\kappa}^{2}}\right)+\frac{1}{\bar{\chi}_{2, m}} \cdot \exp \left(\frac{\tilde{\sigma}_{2}^{2}}{\tilde{\kappa}^{2}}\right)$ and $\mathrm{B}=\frac{1}{\bar{\chi}_{1, m} \cdot \bar{\chi}_{2, m}} \cdot \exp \left[\frac{\tilde{\sigma}_{1}^{2}+\tilde{\sigma}_{2}^{2}}{\tilde{\kappa}^{2}}\right]$.

Using the derivative of (20) in regard to $\chi_{T}$, the PDF of (18) can be rewritten as

$P_{C}(\chi)=\sum_{l=0}^{M}(-1)^{l}\left(\begin{array}{l}M \\ l\end{array}\right) \mathrm{A}^{M-l} \mathrm{~B}^{l}(M+l) \chi^{M+l-1}$.

To attain an exact outage probability (integral-form), (8) is substituted in (15) by using the proper index for hop segments $i=1$ and 2; subsequently (15) is substituted in (19), yielding the exact solution of outage probability, $\tilde{P}_{\text {out }}$, with a non-closed form expression. However, this process was omitted because of its complex integration and high computational load. The problem can be resolved using MATLAB software, as presented in the analytical results in the Section V for the accuracy comparisons. 


\section{BIT-ERROR-RATE (BER) PERFORMANCE}

An analytical approximation of the BER performance was derived using the PDF closed-form in (21) and the MGF [11, 12]. By definition, the average error probability can be expressed using a generalized equation $[11,12]$ :

$$
P_{e}=\int_{0}^{\infty} a Q(\sqrt{b \chi}) P_{C}(\chi) d \chi
$$

where $P_{e}$ is the average error probability in the basis of Gaussian Q-function, $P_{C}(\chi)$ is the PDF derived in (21), and $a$ and $b$ are constant values relative to modulation constellation size $L$ [12]. Hence, the average BER (22) can be rewritten using $[11,12]$

$P_{e}=\frac{a}{\pi} \int_{0}^{\pi / 2} \mathcal{M}_{C}\left(-\frac{b}{2 \sin ^{2} \theta}\right) d \theta$

where $\mathcal{M}_{C}\left(\right.$.) is the MGF and $a=4 / \log _{2} L \cdot(\sqrt{L}-1 / \sqrt{L})$ , $b=3 \log _{2} M /(M-1)$ for L-QAM.

By definition $\left[11\right.$, p. 187] $\mathcal{M}_{C}(-s)=\mathcal{L}\left[P_{C}(\chi)\right]$, is derived as follows:

$$
\begin{aligned}
\mathcal{M}_{C}(-s) & =\sum_{l=0}^{M}(-1)^{l}\left(\begin{array}{l}
M \\
l
\end{array}\right) \mathrm{A}^{M-l} \mathrm{~B}^{l}(M+l) \frac{(M+l-1) !}{(s)^{M+l}} \\
& =\sum_{l=0}^{M}(-1)^{l}\left(\begin{array}{l}
M \\
l
\end{array}\right) \mathrm{A}^{M-l} \mathrm{~B}^{l} \frac{(M+l) !}{(s)^{M+l}}
\end{aligned}
$$

where $\mathcal{L}[$.$] is a Laplace transform and \mathcal{M}_{C}(s)$ is derived using:

$$
\mathcal{M}_{C}(s)=\sum_{l=0}^{M}(-1)^{l}\left(\begin{array}{l}
M \\
l
\end{array}\right) \mathrm{A}^{M-l} \mathrm{~B}^{l} \frac{(M+l) !}{(-s)^{M+l}} .
$$

After substituting (25) in (23) and replacing the variable $s=-b / 2 \sin ^{2} \theta$, the average BER can be written in a closed-form expression as follows:

$$
P_{e}=a \sum_{l=0}^{M}(-1)^{l}\left(\begin{array}{l}
M \\
l
\end{array}\right)\left(\begin{array}{l}
2 M+2 l \\
M+l
\end{array}\right) \mathrm{A}^{M-l} \mathrm{~B}^{l} \frac{(M+l) !}{2^{M+l+1} \cdot b^{M+l}} .
$$

The exact BER expression (integral form) can be processed by substituting (8) in (15) and (15) in (17) for CDF of multirelaying, followed by using a derivative in regard to $\chi$ for a corresponding PDF. Subsequently, the exact BER expression can be obtained using a similar MGF process (Appendix 2). Because this is not a closed form expression, an exact BER analysis must be performed using MATLAB programming subroutines because of the aforementioned computational complexity and procedures. The analytical and simulation results were compared with the closed-form approximation to determine their accuracy and tightness, as presented in Section V.

\section{NUMERCIAL AND SIMULATION RESULTS}

This section provides numerical and simulation results to validate the analytical derivations of the outage probabilities and BER performance levels. The exact formalism (integralform) was treated as a benchmark to corroborate the closedform approximations. In the analyses, dual-hop multirelaying was primarily considered for a semi-asymmetric network (i.e., $\bar{\chi}_{1, m}=\bar{\chi}_{2, m}$ ) and multi-relay nodes $\mathbf{M}=4$. The transmission rate was given as $\lambda=1 \mathrm{bit} / \mathrm{Hz} / \mathrm{sec}$ for all analyses and the constellation size was $L=16$ (i.e., 16QAM) for the BER analysis. The Jakes model [14] was employed to mimic composite fading channels that comprise $2 \mathrm{M}$ complex narrow-band Gaussian random variables; the normalized Doppler frequency $f_{D} T=0.01$ and log-normal fading rate $f_{L} T=0.001$ were set relative to the bit duration $T$. The simulation results were averaged over $10^{6}$ MonteCarlo simulations per SNR value.

\section{A. Outage Analysis}

Figures 2 and 3 show the outage probabilities of the analytical deviation and exact solution compared with the simulation results for balanced shadowing over dual-hop (i.e., $\tilde{\sigma}_{1}=\tilde{\sigma}_{2}$ ), with standard deviations of $4 \mathrm{~dB}$ and $6 \mathrm{~dB}$, respectively. Figure 2 shows the approximation that used the analytical derivation in (20) produced acceptable accuracy by using $\tilde{\sigma}_{1}=\tilde{\sigma}_{2}=4 \mathrm{~dB}$, compared with the exact solution and simulation results for all multi-relaying node numbers; in other words $M=1,2,3$ and 4.

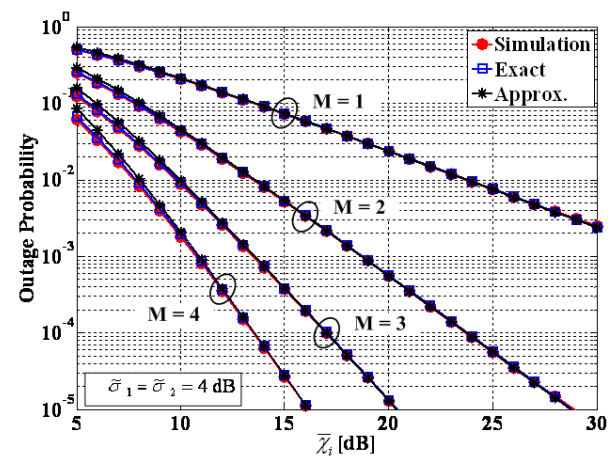

Fig. 2 Outage probability vs. single hop $\bar{\chi}_{i}, i=1,2$ with balanced shadowing relaying hops $\left(\tilde{\sigma}_{1}=\tilde{\sigma}_{2}=4 \mathrm{~dB}\right)$.

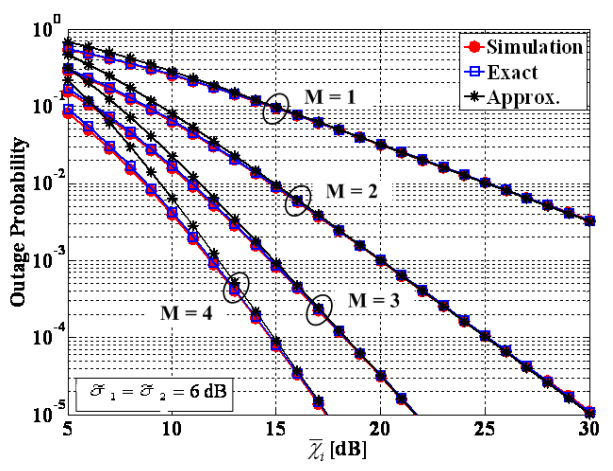

Fig. 3 Outage probability vs. single hop $\bar{\chi}_{i}$ with balanced shadowing relaying hops $\left(\tilde{\sigma}_{1}=\tilde{\sigma}_{2}=6 \mathrm{~dB}\right)$. 
Figure 3 shows that compared with the exact curves and simulation results, the approximations were offset by large standard deviations $\left(\tilde{\sigma}_{1}=\tilde{\sigma}_{2}=6 \mathrm{~dB}\right)$ and a large diversity order (i.e., $\mathrm{M}=4$ ), especially in low regime $\operatorname{SNR}\left(\bar{\chi}_{i} \leq 10\right.$ $\mathrm{dB})$.

Figure 4 shows unbalanced shadowing in a dual-hop relay where $\tilde{\sigma}_{1}=5 \mathrm{~dB}$ and $\tilde{\sigma}_{2}=7 \mathrm{~dB}$. Similar performance curves as those shown in Fig. 3 were achieved. Numerous multirelay nodes (diversity order) achieved considerable power efficiency. However, the difference between the closedform approximation and exact expression increases as the number of relay node increases. The analytical expression performs effectively at an average relaying power of $\bar{\chi}_{i} \geq$ $10 \mathrm{~dB}$, whereas the approximation curve is slightly less accurate for $\bar{\chi}_{i}<10 \mathrm{~dB}$ (i.e., $0.5 \mathrm{~dB}$ difference at outage probability of $10^{-2}$ with $M=4$ ). However, the closed-form approximation considerably reduces the computational load and offers a simple and efficient outage analysis framework.

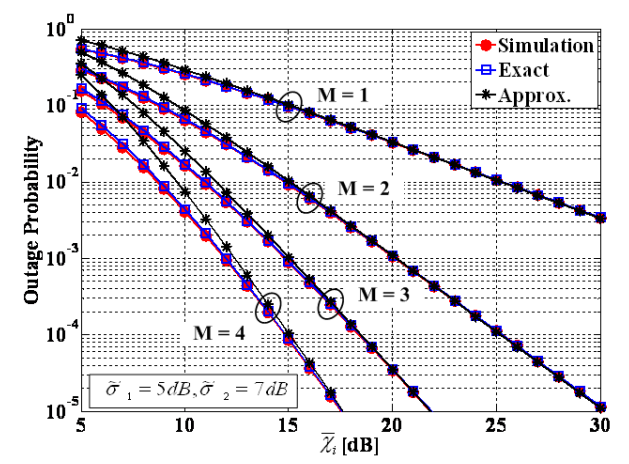

Fig. 4 Outage probability vs. single hop $\bar{\chi}_{i}$ with unbalanced shadowing relaying hops $\left(\tilde{\sigma}_{1} \neq \tilde{\sigma}_{2}\right)$.

\section{B. BER Analysis}

This subsection introduces the average BER performances, which are compared with the exact expression and simulation results for a 16QAM modulating signal. Figures 5 shows that the approximation that employs the analytical derivation in (26) provides acceptable accuracy, whereas the differentiable gap becomes apparent in a low regime of $\chi_{i}$ with large diversity order (i.e., $M=4$ ). However, the analytical derivation is promising and provides acceptable accuracy levels for a difference of less than $1 \mathrm{~dB}$ at $\mathrm{BER}=10^{-2}$, even when $\mathrm{M}=4$.

Figures 6 and 7 show that the diversity benefits of multirelay (M-order) are offset at large standard deviations in any relay hop (i.e., $\tilde{\sigma}_{i} \geq 6 \mathrm{~dB}$ ) when numerous multi-relay nodes (diversity order) are present. Figure 7 indicates that the accuracy can be considerably reduced for large standard deviation occurring at any relay hop components when the proposed analytical derivations are employed. When the number of nodes $(\mathrm{M})$ increases, the probability of achieving a high diversity gain increases; however, the approximation becomes slightly less accurate. Finally, the BER performance levels of the proposed analytical derivation were asymptotically tight with those of the exact solution and simulations when log-normal standard deviation constraints were applied.

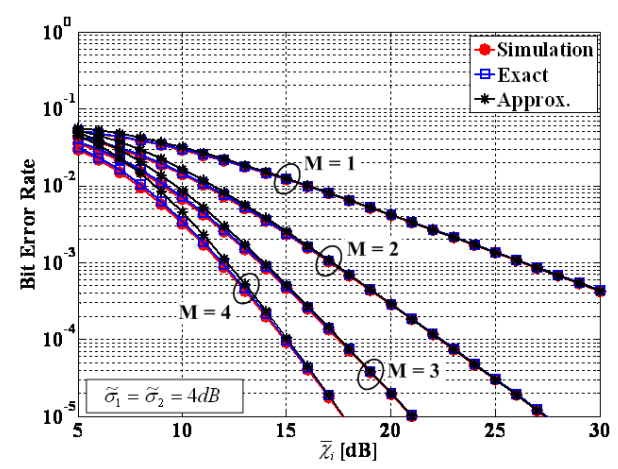

Fig. 5 Average BER on the 16QAM vs. single hop $\bar{\chi}_{i}$ with $\tilde{\sigma}_{1}=\tilde{\sigma}_{2}=4 \mathrm{~dB}$.

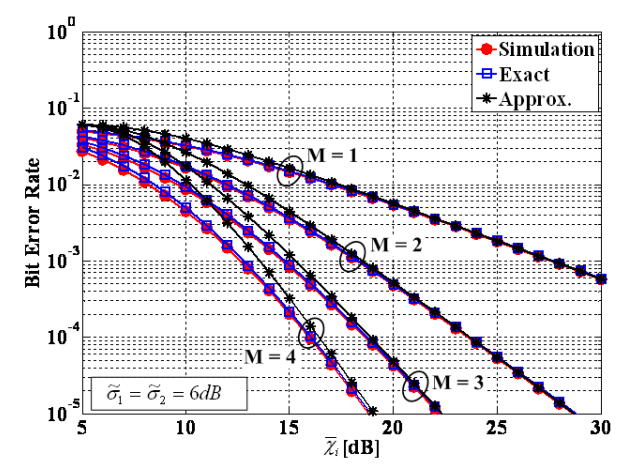

Fig. 6 Average BER on the 16QAM vs. single-hop $\bar{\chi}_{i}$ with $\tilde{\sigma}_{1}=\tilde{\sigma}_{2}=6 \mathrm{~dB}$.

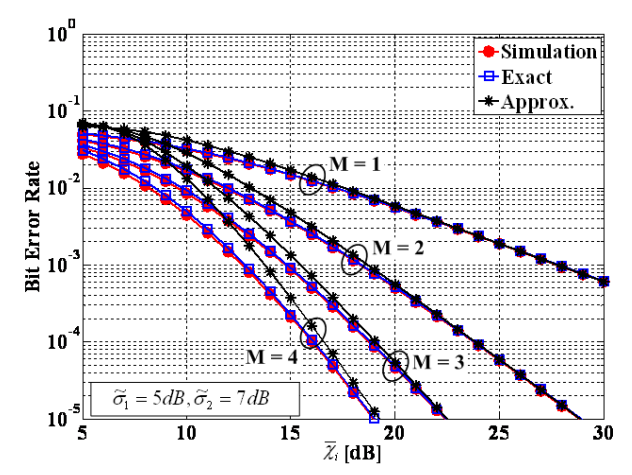

Fig. 7 Average BER on the 16QAM vs. single-hop $\bar{\chi}_{i}$ with $\tilde{\sigma}_{1}=5 \mathrm{~dB}$ and $\tilde{\sigma}_{2}=7 \mathrm{~dB}$.

\section{CONCLUSION}

In this paper, a simple and effective analysis framework was proposed for evaluating the performance levels of a 
dual-hop multi-relaying system over composite fading. In particular, this paper presents analytical derivations of outage probability and BER performance levels, where closed-form expressions comprise the conditional constraint that relaying power is relatively large compared with the target threshold, based on the max-mini relay selection scenario. This relay power constraint consistently meets the performance expectation by using the proposed analytical closed-form expressions, where the low power region loses accuracy. This analysis framework is computationally efficient and promising for evaluating the shadowing effect and diversity gain. The Monte Carlo simulation results and exact solutions were compared regarding outage probability and BER performance levels, respectively; these showed asymptotic tightness to the approximations that used the analytical derivations. In all cases, the accuracy level decreased as the diversity order $\mathrm{M}$ or the standard deviation increased.

\section{REFERENCES}

[1] Mazen O. Hasna and Mohamed-Slim Alouini, "A Performance Study of Dual-Hop Transmission with Fixed Gain Relays," IEEE Transactions on Wireless Communications, Vol. 3, No. 6, November 2004, pp. 1963 - 1968.

[2] Abdulkareem Adinoyi et al., "Performance of Selection Relaying and Cooperative Diversity," IEEE Trans. on Wireless Communications, Vol. 8, No. 12, December 2009, pp. 5790 -5795.

[3] Qijia Liu, Wei Zhang, Xiaoli Ma, and G. Tong Zhou, Designing Peak Power Constrained Amplify-andForward Relay Networks with Cooperative Diversity," IEEE Transactions on Wireless Communications, Vol.11, No. 5, May 2012, pp. 1733 -1743.

[4] Ashkan Kalantari, Mohammadali Mohammadi, and Mehrdad Ardebilipour, "Performance Analysis of Opportunistic Relaying over Imperfect Nonidentical Log-normal Fading Channels," IEEE $22^{\text {nd }}$ PIMRC, 2011, pp.1909-1913.

[5] Gaofeng Pan, Eylem Ekici, and Quanyuan Feng, "BER Analysis of Threshold Digital Relaying Schemes over Log-Normal fading Channels," IEEE Communications Letters, Vol. 15, No. 7, July 2011, pp. $731-733$.

[6] M. Safari, M. Uysal, "Cooperative diversity over log-normal fading channels: performance analysis and optimization," IEEE Transactions on Wireless Communications, Vol.7, No. 5, May 2008, pp. 1963- 1972.

[7] Marco Di Renzo, F. Graziosi, and F. Santucci, “A comprehensive framework for performance analysis of cooperative multi-hop wireless systems over Lognormal fading channels," IEEE Transaction on
Communications, Vol. 58, No. 2, February 2010, pp. 531- 544.

[8] Torabi Mohammad, Wessam Ajib and David Haccoun, "Performance Analysis of Amplify-andForward Cooperative Network with Relay Selection Over Rayleigh Fading Channels, " IEEE VTC Fall 2009, pp.1 -5.

[9] Elzbieta Beres and Raviraj Adve, "Selection Cooperation in Multi-Source Cooperative Networks," IEEE Transactions on Wireless Communications, Vol.7, No.1, January, 2008, pp. 118-127.

[10] A. Papoulis, "Probability, Random Variables, and Stochastic Process," McGraw-Hill, 1991

[11] Andrea Goldsmith, "Wireless Communications", Cambridge University Press, 200.

[12] M. K. Simon and Mohamed-Slim Alouini, "Digital Communication over Fading Channels," John Wiley \& Sons, Inc., 2005.

[13] H. A. David, Order Statistics. New York: John Wiley and Sons Inc., $2^{\text {nd }}$ Ed., 1980.

[14] W. C. Jakes, Microwave Mobile Communications, IEEE Press 1994. 